\title{
Tumor fantasma del pulmón
}

\section{Phantom tumor of the lung}

Dávila-Hernández C, Ñahuis Palomino C, Peralta-Obregón P, BendezúRamos G, J Cárdenas-Trejo, Picoy-Romero D. Tumor fantasma del pulmón. Rev Soc Peru Med Interna. 2020;33(4): I6I-I63. https://doi.org/10.36393/spmi.v33i4.564

\begin{abstract}
RESUMEN
Paciente mujer de 95 años de edad, con disnea nocturna, tos seca y fiebre de $39,2{ }^{\circ} \mathrm{C}$ por seis días y antecedentes de reacción adversa a paracetamol y claritromicina; hipertensa y se controla con losartán. Al examen: PA 100/60 $\mathrm{mm} \mathrm{Hg}$, frecuencia cardiaca 90 latidos/min, frecuencia respiratoria 22 excursiones/min. Edema de miembros inferiores. Pulmones: murmullo vesicular disminuido en ambas bases. Cardiovascular: ruidos cardiacos rítmicos, de baja intensidad. Resto del examen normal. La radiografía de tórax reveló una imagen nodular densa, homogénea, ovalada en el hemitórax izquierdo. La tomografía mostró una efusión pleural en la cisura oblicua izquierda. Se le trató como insuficiencia cardiaca descompensada. Cinco días, la radiografía de tórax no evidenció imagen nodular descrita por lo que se confirmó el diagnóstico de tumor fantasma del pulmón.
\end{abstract}

Palabras clave: tumor fantasma, derrame pleural, insuficiencia cardiaca.

\section{ABSTRACT}

A 95-year-old female patient with nocturnal dyspnea, dry cough and $39.2{ }^{\circ} \mathrm{C}$ fever for six days and a history of adverse reaction to acetaminophen and clarithromycin; hypertensive and controlled with losartan. On examination: PA $100 / 60 \mathrm{~mm}$ $\mathrm{Hg}$, heart rate 90 beats/min, breathing rate 22 excursions/ min. Lower limbs oedema. Lungs: vesicular murmur decreased at both bases. Cardiovascular: rhythmic, low-intensity heart sounds. Rest of the exam was normal. Chest $x$-ray revealed a dense, homogeneous, oval nodular image in the left lung. The CT scan showed a pleural effusion in the left oblique fissure. It was treated as decompensated heart failure. Five days, the chest

Médico internista. Hospital IV Augusto Hernández Mendoza (AHM), EsSalud, Ica. Docente de la Facultad de Medicina Humana Daniel Alcides Carrión, Universidad Nacional San Luis Gonzaga de Ica, Perú.

2 Médico residente de medicina interna. Hospital IV AHM, EsSalud, Ica, Perú.

3 Médico residente de radiología. Hospital IV AHM, EsSalud, Ica, Perú.

4 Médico residente de patología clínica. Hospital IV AHM, EsSalud, Ica, Perú. $x$-ray showed no nodular image described so the diagnosis of phantom tumor of the lung was confirmed.

Keywords: phantom tumor, pleural effusion, heart failure

\section{INTRODUCCIÓN}

En radiología de tórax, el tumor fantasma, tumor evanescente o pseudotumor del pulmón es un derrame pleural encapsulado y localizado en una cisura donde el líquido abomba las dos caras de la pleura visceral creando una imagen redondeada con aspecto de huso, y es interpretada como una manifestación radiológica poco común en personas con falla cardíaca. ${ }^{1}$ Estas lesiones se pueden confundir con infecciones o lesiones tumorales y desaparecen cuando se establece el tratamiento, por lo cual es fundamental realizar un diagnóstico adecuado que evite la práctica de estudios invasivos y no invasivos. ${ }^{2}$

Esta entidad fue descrita en 1928, por H. J. Stewart, tras la realización de una autopsia para confirmar una sospecha clínica y la denomino "hidrotórax lobar". ${ }^{3,4}$ Por otra parte, hay que tener presente que esta expresión radiográfica de 
la insuficiencia cardíaca no es usual y es benigna, pero la enfermedad de base es muy frecuente y presenta una tasa muy alta de mortalidad. ${ }^{5}$

\section{PRESENTACIÓN DEL CASO}

Paciente mujer de 95 años de edad, traída por hija a la emergencia del hospital IV Augusto Hernández Mendoza (AHM), EsSalud, Ica, por presentar dificultad para respirar en los últimos seis días, que se exacerba por las noches, disminuye cuando "se sienta" y se acompaña de tos seca que se presenta esporádicamente; así mismo, ha presentado en los dos últimos fiebre de $39,2^{\circ} \mathrm{c}$ que cede con Antalgina $500 \mathrm{mg}$ y paños de agua tibia.

Antecedentes: reacción adversa a paracetamol, claritromicina; hipertensión arterial, toma losartan $50 \mathrm{mg}$ al día; cirugías anteriores: apendicetomía y colecistectomía.

Al examen físico: PA 100/60 mm Hg, FC 90 lat $/ \mathrm{min}$, FR $22 \mathrm{resp} / \mathrm{min}$. Edema de miembros inferiores $1+/ 3+$. Pulmones: murmullo respiratorio disminuido en base de ambos hemitórax. CV: ruidos cardiacos rítmicos, de baja intensidad. El resto del examen sin alteraciones significativas.

Exámenes auxiliares. Hemograma: leucocito: 8 300/ mm3; Hb 11,6 g/dl, plaquetas 182/mm3. Glucosa 119 mg/ $\mathrm{dl}$, creatinina $0,71 \mathrm{mg} / \mathrm{dl}$, sodio $134 \mathrm{mmol} / \mathrm{L}$, potasio 3,6 mmol/L. Sedimento urinario: leucocitos 15-20/campo, hematíes 0-1/ campo.

Radiografía de tórax: imagen nodular densa, homogénea, ovalada en hemitórax izquierdo. (Figura 1)

El hallazgo radiológico determinó su hospitalización en el servicio de medicina interna para estudios, y se inició tratamiento antibiótico para infección respiratoria y del tracto urinario.

Otros resultados: fibrinógeno $329 \mathrm{mg} / \mathrm{dl}$, TGO: 43,1 U/L, TGP: 20,8 U/L, fosfatasa alcalina: $93 \mathrm{u} / \mathrm{L}$, gamma glutamil transpeptidasa $29 \mathrm{U} / \mathrm{L}$, proteínas totales $5,67 \mathrm{~g} / \mathrm{dl}$, albumina $3,17 \mathrm{~g} / \mathrm{dl}$. Tiempo de protrombina $12,2 \mathrm{seg}$, INR 0,9.

Tomografía torácica: parénquima pulmonar de transparencia conservada, calcificaciones de la pared de la aorta torácica,

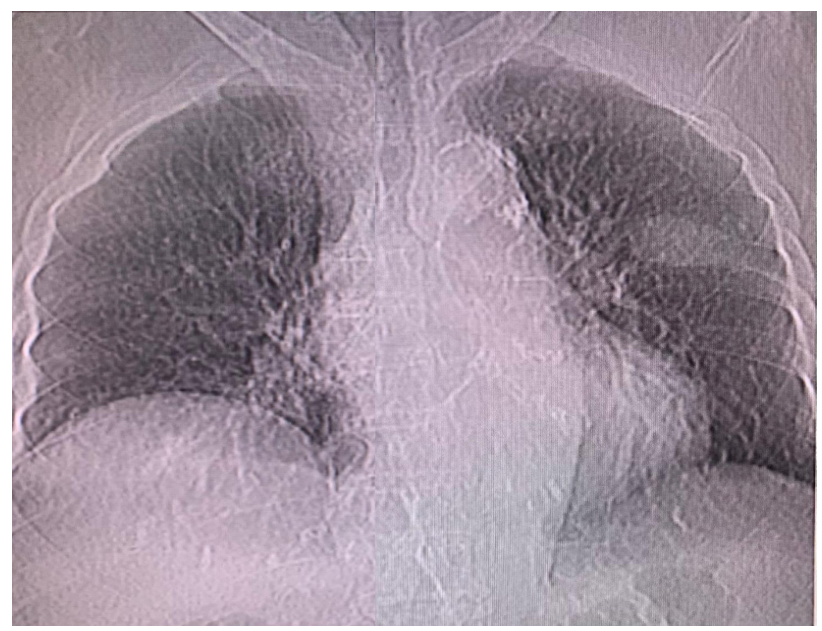

Figura I. Radiografía de tórax al ingreso, con masa ovoidea en el hemitórax izquierdo. contenido de densidad líquida en volumen aproximado 9,5 $\mathrm{ml}$ en cisura oblicua izquierda en relación a efusión pleural encapsulada; en conclusión: efusión pleural en cisura oblicua izquierda, angioesclerosis de aorta torácica (Figura 2).

Marcadores tumorales: CEA: 3,21 ng/ml (VN: $<5 \mathrm{ng} / \mathrm{ml}$ ), AFP: $2,96 \mathrm{ng} / \mathrm{ml}(\mathrm{VN}:<10 \mathrm{ng} / \mathrm{ml}), \mathrm{CA} 125: 29,61 \mathrm{U} / \mathrm{ml}$ (VN: < $35 \mathrm{U} / \mathrm{ml}), \mathrm{CA} 19,9: 24,05 \mathrm{U} / \mathrm{ml}(\mathrm{VN}:<35 \mathrm{U} / \mathrm{ml})$, CA 15,3: 10,49 U/ml (VN: < $35 \mathrm{U} / \mathrm{ml})$.

Urocultivo: positivo a Escherichia coli, recuento de colonias: $+100000 \mathrm{ufc} / \mathrm{ml}$, sensible a ceftriaxona.

Se inició tratamiento para insuficiencia cardiaca descompensada con furosemida, carvedilol, espironolactona. Cinco días después no se evidenció la imagen nodular, lo que determina el diagnóstico de tumor fantasma del pulmón (Figura 3).

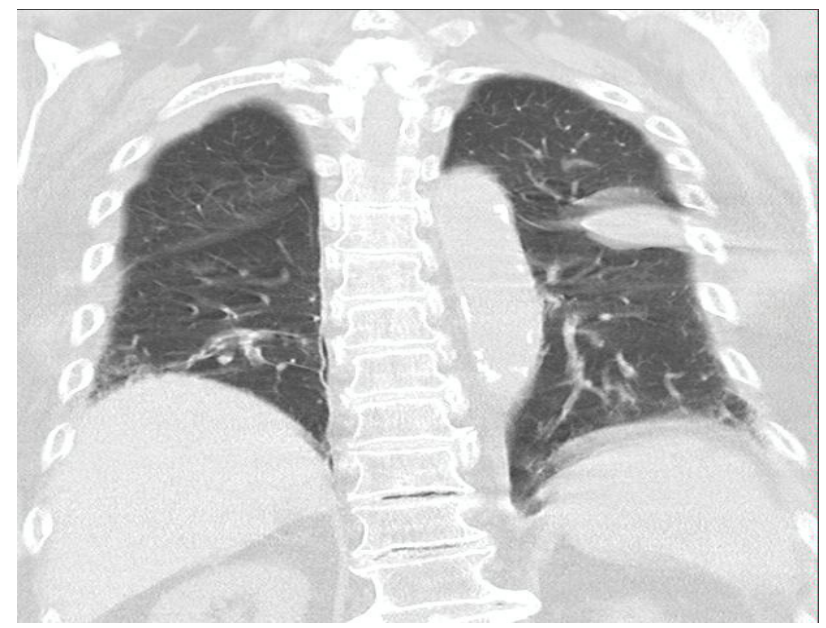

Figura 2. Masa ovoide intercisural izquierda.

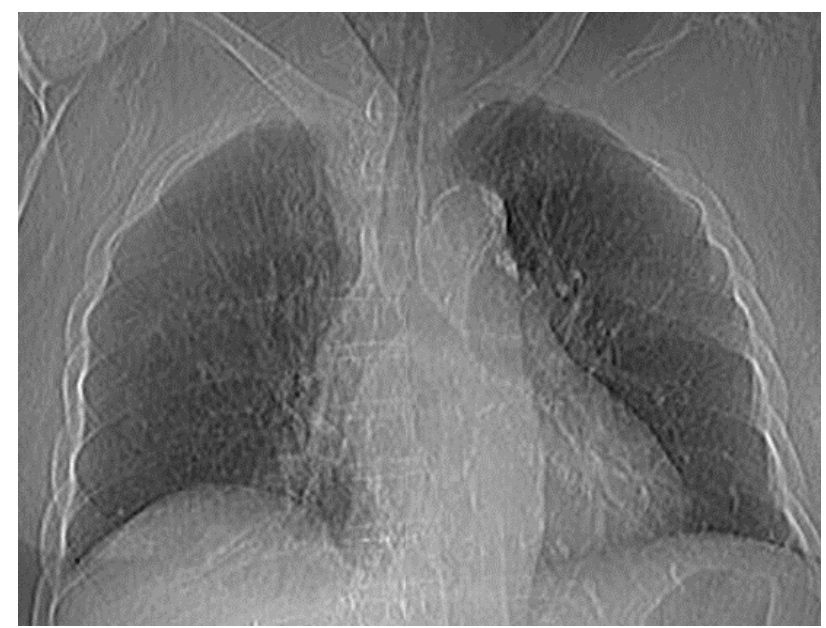

Figura 3. Radiografía de tórax cinco días después del ingreso, con desaparición de la imagen ovoidea.

\section{DISCUSIÓN}

La pleura es una membrana serosa compuesta de células mesoteliales y tejido conectivo laxo, y se divide en pleura parietal y visceral que delimitan el espacio pleural, el cual 
contiene 10-15 ml de líquido para facilitar el desplazamiento de las mismas. No existe comunicación entre el lado derecho y el izquierdo. La nutrición vascular de la pleura parietal se hace a través de la circulación sistémica y la visceral a través de la bronquial y pulmonar.

En condiciones normales, la pleura no es visible en una radiografía de tórax. El tumor fantasma es un derrame pleural encapsulado localizado en una cisura que suele verse con más frecuencia en los pacientes con insuficiencia cardiaca. ${ }^{6}$ La incidencia de este hallazgo es desconocida debido a que sólo se basa en reportes de casos, por lo cual se ha considerado como una manifestación radiológica infrecuente. $^{7}$ La etiopatogenia de esta patología no se conoce con claridad, clásicamente se postula que podría deberse a defectos pleurales congénitos o adherencias secundarias a procesos inflamatorios previos. ${ }^{8}$

Los tumores fantasmas surgen siempre que la trasudación del espacio vascular pulmonar excede la capacidad de resorción de los linfáticos pleurales. ${ }^{9}$ La exploración física de estos pacientes no hace sospechar la existencia de estas colecciones, solo mediante la radiografía de tórax podemos determinar el derrame en poca cantidad confinado a una de las cisuras pulmonares. Se presentan predominantemente en hombres y en el hemitórax derecho, más frecuente en la cisura transversal derecha y con menos frecuencia en la cisura oblicua, mientras que en el hemitórax izquierdo se describen esporádicamente. ${ }^{10} \mathrm{El}$ diagnóstico diferencial se realiza con otras enfermedades que puedan provocar alteraciones radiológicas similares: tumores pulmonares, metástasis, neumonías, tuberculosis, infartos pulmonares o quistes hidatídicos. ${ }^{11}$ En nuestro caso, la primera opción fue una tumoración pulmonar pero los marcadores tumorales fueron normales y la tomografía torácica alejó la posibilidad de alguna neoplasia. En el caso de neumonía redonda, esta predomina en la infancia, se acompaña de leucocitosis y es un proceso raro en adultos. 12

La importancia de este caso radica en describir la dificultad del diagnóstico del tumor fantasma y la necesidad de un enfoque adecuado para abordarlo con miras a optimizar el tiempo de intervenciones diagnósticas y terapéuticas, y por la escasa información reportada sobre este tema en nuestro medio.

\section{REFERENCIAS BIBLIOGRÁFICAS}

I. Sánchez C, Pedrona A, Casanova R. Patología pleural. En: Ed. McCraw-Hill. Diagnóstico por imagen. Tratado de radiología clínica. Barcelona, 1997:395-396.

2. Feldman DJ. Localized interlobarpleural effusion in heart failure.JAm Med Assoc. 1951; 146: 1408-9.

3. Stewart HJ. Pleural effusion localized in an interlobar space: Report of a case of heart failure together with autopsy. American Heart Journal. 1928;4:227-35.

4. Llens Pagès A, Fitó Tarragó R, Moret Serralta A. Tumor fantasma. FMC. 20I0; I 7:501-502.

5. Hupcey JE, Penrod J, Fogg J. Heart failure and palliative care: implications in practice. J Palliat Med. 2009; I2:53 I-6. URL disponible en: http://dx.doi.org/I0.1089/jpm.2009.0010

6. Sureka B, Thukral BB, Mittal MK, Mittal A, Sinha M. Radiological review of pleural tumors. Indian J Radiol Imaging. 20I3; 23:3। 3

7. Weiss W, Boucot KR, Gefter WI. Localized interlobar effusion in congestive heart failure. Ann Intern Med. 1953;38: I I77-86.

8. Millard CE. Vanishing of phantom tumor of the lung; localized interlobar effusion in congestive heart failure. Chest. 1971;59:675-7.

9. FG Fleischner. Disposición atípica del derrame pleural libre. Radiologic Clinics of North America. 1 963; | :347-362.

10. KP Buch y RS Morehead. Múltiples tumores desaparecidos del lado izquierdo. Chest. 2000; I |8(5) | 486-89.

II. Repiso M EM, Jiménez F, Barrón M. Un caso de tumor fantasma. SEMERGEN. 2001;27:320-I.

12. M. Zylberman, E. Cordova, G. Farace. Round pneumonia in adults, an unusual presentation of lung parenchymal infection: a report of two cases and review of the literature. Clin Pulm Med. 2006; I 3: 229231 .

\section{CORRESPONDENCIA}

Carlos Alberto Dávila-Hernández

dh39130@hotmail.com

Fecha de recepción: 06-09-2020.

Fecha de aceptación: |6-| |-2020. 